\title{
Studies on a Radio-resistant Coccus Isolated from Bombay Duck (Harpodon nehereus)
}

\author{
By N. F. LEWIS \\ Biochemistry and Food Technology Division, Bhabha Atomic Research Centre, \\ Trombay, Bombay 85, India \\ (Accepted for publication 3 February 197I)
}

\begin{abstract}
SUMMARY
An extremely radio-resistant orange-red pigmented Micrococcus sp. was isolated from irradiated Bombay duck (Harpodon nehereus). The organism was a Grampositive vegetative tetracoccus, catalase-positive, sensitive to heat and nonpathogenic. It differed from $M$. radiodurans by being smaller in size, salt-tolerant, hydrolysing gelatin very slowly, and much more resistant to gamma radiation. The radiation survival curve of the new coccus was sigmoidal, punctuated with a very large shoulder extending beyond I.5 Mrad.
\end{abstract}

\section{INTRODUCTION}

Micrococcus radiodurans, isolated originally by Anderson et al. (I956) from meat exposed to $4 \mathrm{Mrad}$, has evoked considerable attention because of its unique radio resistance. Ever since its isolation this organism has served as a dynamic model for studies in mechanisms of radio resistance in bacteria. The radio resistance of $M$. radiodurans has been postulated to be due to pigmentation (Moseley, I963; Krabbenhoft, Anderson \& Elliker, 1967), the presence of mercaptoalkylamine (Anderson et al. 1959), intracellular radio-protective compounds (Bruce, 1964), the ultrafine structure of the cell wall (Thornley, Horne \& Glauert, 1965) and to fatty-acid composition (Lewis, 1965). However, the work of Setlow \& Duggan (1964) and more recently of Moseley \& Laser (1965) implies that the high radio resistance of $M$. radiodurans may be attributed to enzyme repair mechanisms for damaged deoxyribonucleic acid (DNA). Micrococcus radiodurans has been subsequently isolated from other sources (Krabbenhoft, Anderson \& Elliker, 1965). Two other radio-resistant Micrococcus species, morphologically similar to $M$. radiodurans, have since been reported - from irradiated haddock fillets (Davis, Silverman \& Masurovsky, 1963) and an aerial contaminant (Murray \& Robinow, 1958).

It was therefore of interest to investigate if this organism was universally present; and, if so, whether it occurred in irradiated tropical fish varieties. During investigations on the extension of the shelf-life of Bombay duck (Harpodon nehereus) using gamma radiation, glistening orange-red pigmented colonies in Petri dish cultures were consistently isolated from fillets exposed to $\mathrm{I} \cdot \mathrm{O}$ to $2 \cdot 0 \mathrm{Mrad}$. When this organism was examined for radiation resistance, cultural, morphological and biochemical characteristics, it was observed that the isolate was distinct from Micrococcus radiodurans or any other Micrococcus species listed in Bergey's Manual, suggesting that it might be a new species. Pure cultures were found to be more resistant to gamma radiation than any microbial species so far reported. The newly isolated coccus was designated $\mathrm{RBD}$, denoting a radio-resistant micro-organism obtained from Bombay duck. 
This culture has been deposited with the National Collection of Type Cultures, Colindale, London.

\section{METHODS}

Cultures. Micrococcus radiodurans, obtained from Professor A. W. Anderson, Oregon State University, Oregon, U.S.A., which was used for comparative purposes throughout this investigation, was cultured in TGYM medium containing $0.5 \%$ tryptone, $0.1 \%$ glucose, $0.3 \%$ yeast extract and $50 \mathrm{mg} . \%$ DL-methionine.

Microbial evaluation of Bombay-duck fillets subjected to $2 \cdot 0 \mathrm{Mrad}$ indicated that only orange-red pigmented cocci survived the radiation treatment. The original isolate was cultured on nutrient agar and subsequently maintained on TGYM agar slants.

\section{Growth characteristics}

Effect of media on growth stimulation was investigated with nutrient agar, TGYM (tryptone, $0.5 \%$; glucose, $0.1 \%$; yeast extract, $0.5 \%$ ), tryptone glucose extract (TGE) agar, as well as TGY agar incorporated with DL-methionine (50 mg. \%) or Bacto Casamino acids $(0.5 \%)$. Growth was evaluated by streaking the surface of deep agar slants and observing periodically over a 6 day incubation period at room temperature $\left(28^{\circ}\right)$. The effect of DLmethionine (essential for propagation of Micrococcus radiodurans) on growth was investigated by incorporating varying concentrations from $20 \mathrm{mg}$. to $2 \mathrm{~g}$./1. of TGY broth. The effect of $\mathrm{pH}$ on growth was ascertained by culturing the organisms in TGYM broth at $\mathrm{pH}$ levels from 5.5 to 9.5 . Tolerance of $\mathrm{NaCl}$ up to a concentration of $7 \%$ in TGYM broth was also investigated. For optimal growth-temperature experiments, inoculated slants were incubated at $15^{\circ}, 28^{\circ}, 37^{\circ}$ and $45^{\circ}$ and growth was assessed by visual examination.

Biochemical tests. Several biochemical tests (Harrigan \& McCance, 1966) were done, including catalase formation, hydrogen sulphide production, gelatin liquefaction, indole production, nitrate reduction, and both acid and gas production from carbohydrates. The inocula for all tests were taken from $24 \mathrm{~h}$. TGYM broth cultures.

The catalase test was performed by adding I ml. culture to I ml. of Io vol. $\mathrm{H}_{2} \mathrm{O}_{2}$ and looking for effervescence within $5 \mathrm{~min}$.; $\mathrm{H}_{2} \mathrm{~S}$ production was demonstrated on triple sugar iron agar slants; gelatin liquefaction was assessed with nutrient gelatin stabs incubated for 30 days at $25^{\circ}$; indole production in I \% tryptone solution was detected by Kovac's indole reagent. Reduction of nitrate was demonstrated after maximal growth was attained $(72 \mathrm{~h}$.). The basal medium for fermentation of carbohydrates was $\mathrm{I} \%$ peptone water incorporating Andrade's indicator for detection of acid produced. Sugars were heat-sterilized as $10 \%$ solutions and used at a final concentration of $\mathrm{I} \%$. Morphological characteristics were studied by taking a loopful of culture on a coverslip, drying in air, inverting over a drop of lactophenol on a glass slide and examining under phase contrast.

Toxin assay. Twenty-four $\mathrm{h}$. and 7 day TGYM broth cultures were examined for pathogenicity. After centrifugation I ml. samples of supernatants and sonicated cell suspensions were injected intraperitoneally into mice which were observed for 8 days. Six mice were used for each sample.

\section{Irradiation}

The radio-resistant coccus RBD and Micrococcus radiodurans were cultured for 40 to $44 \mathrm{~h}$. at room temperature, in shake flasks containing TGYM broth $\left(\mathrm{pH}_{7 \cdot 2}\right)$. The populations were then standardized to a density of approximately $10^{8}$ bacteria $/ \mathrm{ml}$. by extinction measure- 
ments, and $5 \mathrm{ml}$. suspensions were dispensed in $9 \times \mathrm{I} \mathrm{cm}$. test tubes, placed in a beaker containing comminuted ice and irradiated within $30 \mathrm{~min}$. in a Gamma-cell 220 (A. E. C. L., Canada) at a dose rate of I I $\mathrm{Krad} / \mathrm{min}$. Temperature during irradiation was maintained at $4^{\circ}$ to $6^{\circ}$. Radiation survivors were recovered on TGYM agar. Colonies were counted after 5 days incubation at room temperature.

\section{RESULTS}

By phase-contrast microscopy the new isolate was seen to be a Micrococcus species morphologically similar to Micrococcus radiodurans (P1. I, fig. I, 2). The organisms occurred predominantly as tetrads, and occasionally in pairs or singly. However, the organism was smaller than $M$. radiodurans, being spheres averaging $\mathrm{I} \cdot 5 \pm 0.2 \mu \mathrm{m}$. in diameter as compared with $2.4 \pm 0.46 \mu \mathrm{m}$. for $M$. radiodurans. Microscopic examination of cultures at different physiological ages did not reveal any over-size cells; $M$. radiodurans cultures normally contained a small percentage of very large cells.

The new coccus always stained Gram-positive, unlike older cultures of Micrococcus radiodurans, which were reported as Gram-variable (Anderson et al. 1959). Growth obtained on TGYM agar slants after $72 \mathrm{~h}$. incubation at $30^{\circ}$ was mucoid, raised glistening orange-red as compared with dull-red growth of $M$. radiodurans. Moreover, unlike $M$. radiodurans the pigment did not diffuse into the agar. Growth rate and pigmentation were greater on TGY agar than on TGE agar where the growth was poor, or on nutrient agar which barely supported growth. TGY agar with DL-methionine (50 mg. \%) or supplemented with $0.5 \%$ Casamino acids (acid-hydrolysed casein) did not change growth or pigmentation. Krabbenhoft et al. (1967) reported greater yields but less pigmentation in cells of $M$. radiodurans grown in TGY medium supplemented with $0.5 \%$ tryptic digest of casein (NZ-case). Use of deionized water for media preparation resulted in poor growth as compared with tap water where both growth rate and yield were considerably enhanced, indicating trace-metal requirement in growth media. Growth was greatly stimulated by vigorous aeration. DL-Methionine, said to be an essential amino acid for growth of $M$.radiodurans (Raj et al. 1960), did not enhance growth of RBD up to a level of $400 \mathrm{mg}$./1.; higher concentrations reduced the yield.

Table I indicates the pronounced differences produced in the growth characteristics of Micrococcus radiodurans and $\mathrm{RBD}$ by altering the $\mathrm{pH}$ of the growth media: $M$. radiodurans had optimum growth at acid $\mathrm{pH}(5.5$ to 6.5$)$ and grew well up to $\mathrm{pH} 8.5$, above which growth was markedly inhibited, while RBD did not grow at $\mathrm{pH} 6.0$ or below but grew well up to pH 9.5. The salt tolerance of these two micro-organisms is recorded in Table 2. The isolate RBD grew well in media containing $\mathrm{NaCl}$ up to $3 \%$ and moderately in media containing $5 \%$ $\mathrm{NaCl} ; M$. radiodurans was inhibited at $2 \% \mathrm{NaCl}$. Temperature affected both organisms similarly. The optimum was $30^{\circ}$ to $37^{\circ}$, while growth was slow at $20^{\circ}$ and nil at both $10^{\circ}$ and $45^{\circ}$.

Both Micrococcus radiodurans and RBD possessed high catalase activity but did not reduce nitrate nor produce indole from tryptophan. While $M$. radiodurans liquefied gelatin within $48 \mathrm{~h}$., no liquefaction was obtained with RBD cultures up to 24 days incubation at $25^{\circ}$; after this, liquefaction proceeded at a very slow rate. Anderson et al. (1956) reported that samples of beef seeded with $M$. radiodurans underwent spoilage which was characterized by considerable proteolysis and $\mathrm{CO}_{2}$ production. However, no proteolysis or gas production could be detected in sterile fish homogenates inoculated with RBD cells and incubated for $48 \mathrm{~h}$. at $30^{\circ}$. The results of the carbohydrate fermentation reactions are listed in Table 3. The new isolate readily produced acid from glucose, fructose and mannitol and gave variable results with 
lactose and galactose; $M$. radiodurans did not produce acid from any of the carbohydrates used. Neither organism produced gas from any of the carbohydrates examined.

Results of the toxin assay indicated that both Micrococcus radiodurans and RBD were non-pathogenic.

A comparison of the radiation resistivity of $\mathrm{RBD}$ and Micrococcus radiodurans is presented in Fig. I. The survival curve of RBD is similar to that of $M$. radiodurans in being sigmoidal but is easily distinguished by a very large shoulder extending beyond $\mathrm{I} \cdot 5 \mathrm{Mrad}$. A radiation dose of $\mathrm{I} \cdot 6 \mathrm{Mrad}$, which sufficed to reduce the viable cell population of $M$. radiodurans to about one-millionth, resulted in only tenfold inactivation of RBD cell suspensions. A dose of 3.2 Mrad was necessary to reduce the viable cell count of RBD to a millionth.

Table I. Effect of $p H$ on growth of Micrococcus radiodurans and $\mathrm{RBD}$

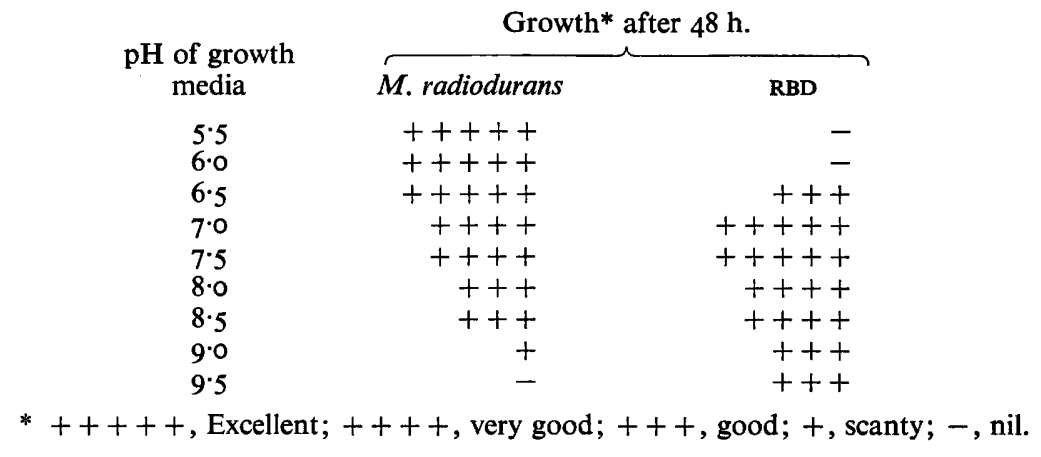

Table 2. Salt tolerance of Micrococcus radiodurans and $\mathrm{RBD}$

$\begin{array}{ccr}\begin{array}{c}\text { NaCl concentra- } \\ \text { tion in growth } \\ \text { media }\end{array} & \overbrace{\text { M. radiodurans }} & \text { Growth* after } 48 \mathrm{~h} . \\ 0 \% & +++++ & +++++ \\ 1 \% & ++++ & +++++ \\ 2 \% & - & +++++ \\ 3 \% & - & +++++ \\ 5 \% & - & +++ \\ 7 \% & \text { SBD } & - \\ & \text { Symbols as in Table I. }\end{array}$

Table 3. Production of acid and gas during carbohydrate utilization by Micrococcus radiodurans and $\mathrm{RBD}$

$\begin{array}{lcccc}\text { Carbohydrate (I \%) } & \overbrace{\text { Acid }}^{\text {M. radiodurans }} & \text { Gas } & \overbrace{\text { Acid }} & \text { Gas } \\ \text { Arabinose } & - & - & - & - \\ \text { Fructose } & + & - & - & - \\ \text { Galactose } & \text { Var } & - & - & - \\ \text { Glucose } & + & - & - & - \\ \text { Glycerol } & - & - & - & - \\ \text { Inositol } & - & - & - & - \\ \text { Lactose } & \text { Var } & - & - & - \\ \text { Mannitol } & + & - & - & - \\ \text { Rhamnose } & - & - & - & - \\ \text { Sucrose } & - & - & - & - \\ \text { Xylose } & - & - & & \end{array}$




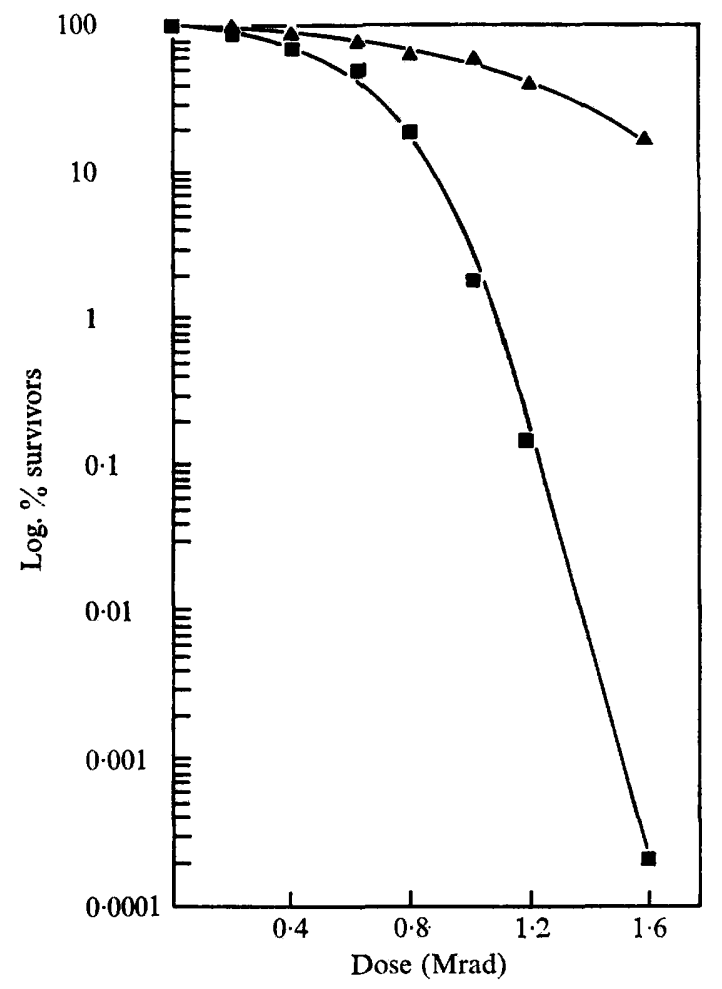

Fig. I. Percentages of RBD (A) and Micrococcus radiodurans ( $\mathbf{(})$ surviving different doses of gamma radiation.

\section{DISCUSSION}

Preliminary studies on cellular components of the new Micrococcus species, which is more resistant to gamma radiation than Micrococcus radiodurans by several orders of magnitude, indicate that besides the evident possession of an extremely efficient enzyme repair system, other factors like pigments and lipids could presumably have contributed to its exceptional radio resistance. The isolation of a new radio-resistant Micrococcus sp. provides further impetus towards establishing mechanisms that may clarify the unique radio resistivity of these organisms.

I wish to thank Dr U. S. Kumta for helpful suggestions during review of the manuscript.

\section{REFERENCES}

Anderson, A. W., Nordan, H. C., Cain, R. F., Parrish, G. \& Duggan, D. (i956). Studies on the radioresistant Micrococcus. I. The isolation, morphology, cultural characteristics and resistance to gamma radiation. Food Technology 10, 575-577.

Anderson, A. W., Raj, H. D., Wang, C. H., Duryee, P. L. \& Elliker, P. R. (1959). Carbohydrate catabolism of a radiation-resistant bacterium. Bacteriological Proceedings, p. 20.

BRUCE, A. K. (1964). Extraction of the radio-resistant factor of Micrococcus radiodurans. Radiation Research 22, I 55-164. 
Davis, N. S., Silverman, G. J. \& Masurovsky, E. B. (1963). Radiation-resistant, pigmented coccus isolated from haddock tissue. Journal of Bacteriology 86, 294-298.

HARRIGAN, W. F. \& MCCANCE, M. E. (1966). Laboratory Methods in Microbiology, pp. 51-68. London: Academic Press.

Krabbenhoft, K. L., Anderson, A. W. \& Elliker, P. R. (1965). Ecology of Micrococcus radiodurans. Applied Microbiology 13, I030-1037.

Krabbenhoft, K. L., Anderson, A. W. \& Elliker, P. R. (1967). Influence of culture media on the radiation resistance of Micrococcus radiodurans. Applied Microbiology 15, 178-185.

LEWIS, N. F (1965). Studies on the Fatty Acid Composition of Micrococcus radiodurans. M.S. thesis, Oregon State University, Corvallis, Oregon.

Moseley, B. E. B. (1963). The variation in X-ray resistance of Micrococcus radiodurans and some of its less pigmented mutants. International Journal of Radiation Biology 6, 489.

Moseley, B. E. B. \& LASER, H. (1965). Repair of X-ray damage in Micrococcus radiodurans. Proceedings of the Royal Society 162, $210-222$.

Murray, R. G. E. \& Robinow, C. F. (I958). International Congress for Microbiology 7 (Abstracts), 427.

Raj, H. D., Duryee, F. L., Deeney, A. M., Wang, C. H., Anderson, A. W. \& Elliker, P. R. (I960). Utilization of carbohydrates and amino acids by Micrococcus radiodurans. Canadian Journal of Microbiology 6, 289-298.

SETLow, J. K. \& DugGan, D. E. (1964). The resistance of Micrococcus radiodurans to ultraviolet radiation. I. Ultraviolet-induced lesions in the cell's DNA. Biochimica et biophysica acta 87, 664-668.

Thornley, M. J., Horne, R. W. \& Glauert, A. M. (I965). The fine structure of Micrococcus radiodurans. Archives of Microbiology 51, 267-289. 


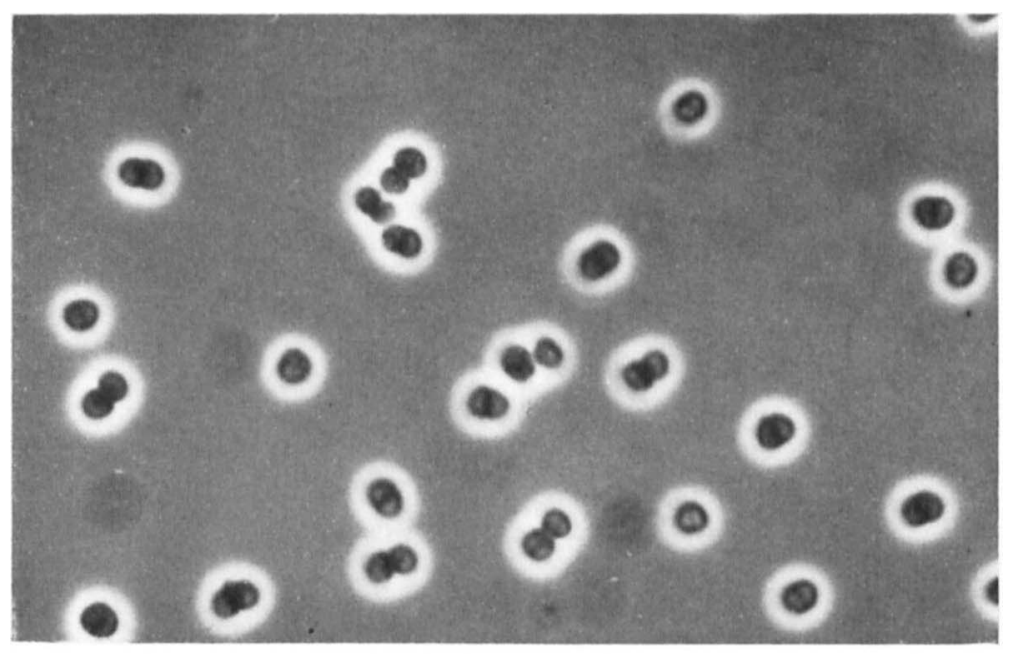

Fig. I

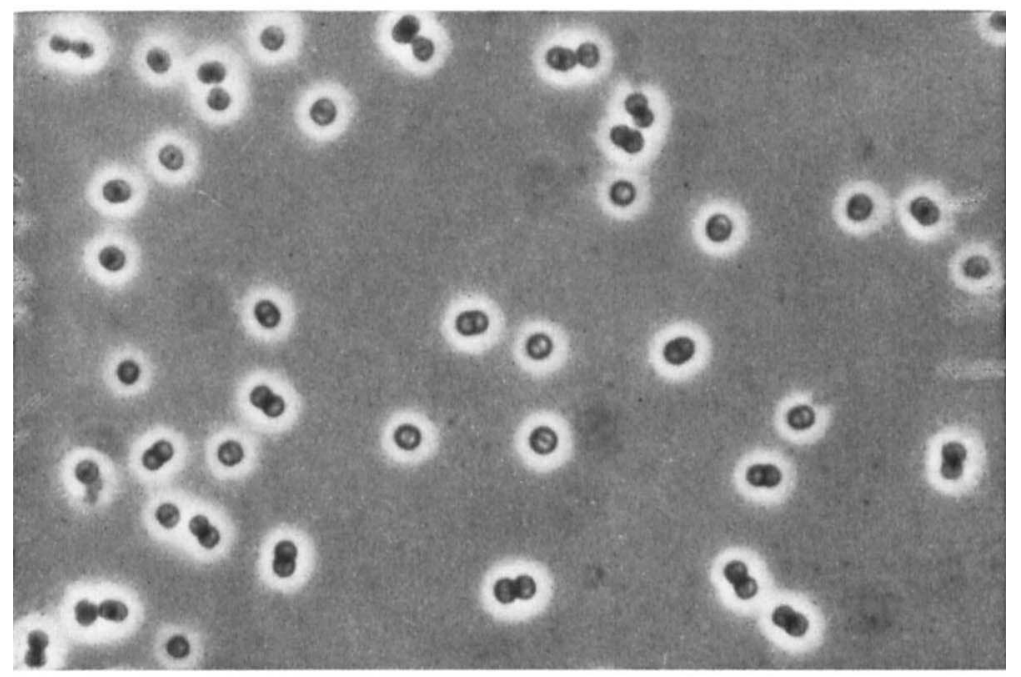

Fig. 2

Plate I

Phase-contrast photomicrographs of Micrococcus radiodurans (Fig. I) and RBD (Fig. 2) in shake cultures. 